\title{
Jamming method based on dynamical convolution against ISAR
}

\author{
†Zhong-Gan Bai, Xing-Song Deng, Wen-Jun Xu and Kun Shu \\ Yangzhou Marine Electronic Instruments Institute, Yangzhou, 225001, China \\ $\dagger$ E-mail: baiy723@sohu.com
}

\begin{abstract}
A new active jamming method based on dynamical convolution against Inverse Synthetic Aperture Radar (ISAR) is set up in this paper. The traditional scatter jammer is noncooperative with the dispenser's gesture, which makes ISAR easy to identify it. The new jamming strategy improves it in two steps. The $1^{\text {st }}$ step is to establish fixed jamming templates of targets using turntable images of the missile model. The $2^{\text {nd }}$ step is to compose the information of the dispenser's gesture by interpolation between adjacent templates at fixed angles of look of sight (LOS).The performance of the new method is analyzed that it can produce the jamming effect changing dynamically both in onedimensional range profiles and in 2-D images during the ballistic full. Simulation is given to show the effectiveness of the method.

Keywords: convolution jamming; ISAR imaging; radar ECM; strategic missile penetration.
\end{abstract}

\section{Introduction}

As an important sensor in ballistic missile defense system, the inverse synthetic aperture radar (ISAR) can reconstruct range profiles (1-D) and two dimensional (2-D) images of moving missiles, which can be used for target detection, classification and identification [1]-[2].Therefore, active jamming technology against ISAR is very important for strategic missile penetration .As noise jamming requires more power, deceive jamming is an important direction in ISAR ECM area.

Few were reported on ISAR ECM because of its sensitivity in defense area. Among them, the jamming method [3]-[5] based on micro-Doppler effect is viewed. A kind of phase modulated jamming technology [6] based on Msequence is researched. A similar clutter jamming (SCJ) method and an digital false-target image synthesiser for countering ISAR are researched [7]-[8].These are of theoretical significance, but have little practical value. In this paper, we will present a new jamming method based-on dynamical convolution, which makes the changing template of the target rapidly during the trajectory full. 
The ISAR imaging principle is viewed in part I. The jamming method based on dynamical convolution is discussed in part II. The performance of the method is analyzed in part III.

\section{ISAR Image Principle}

The research of ISAR imaging is based on the theory that the total electromagnetic scattering can be equivalent to the sum of the electromagnetic scatterings at some local positions of the target, which can be proven in theory and experiment. In a certain area of the rotating angle, non-cooperative moving target can be equivalent to the uniform moving rotary table target, so the target can be equivalent to the sum of the electromagnetic scatterings at some local positions of the target. Meanwhile, the positions and the intensities of the I scatters of the target change little among the imaging aperture. The 1-D range profiles and 2-D image of the target is shaped when the stretch processing makes the signal bandwidth small and the motion compensation is finished, among which the Range-Doppler algorithm is usually applied.

\section{Dynamical Convolution against ISAR}

\subsection{Generation of the algorithm}

Ignore the minor distance between the scatter jammer and the target, which is to be protected, the target echo denotes as,

$$
s(t)=\exp \left[j 2 \pi\left(f_{0} t+K t^{2} / 2\right)\right]
$$

Let the missile be one point target, the rcs of the missile be $\sigma$,so its response function is,

$$
c(t)=\sigma \cdot \delta(t-\tau)
$$

And the echo of the missile is,

$$
p(t)=c(t) * s(t)
$$

The jammer convolute the received signal with the video function $f(t)$, and then re-transmit it out.

$$
J(t)=f(t) * s(t)(4)
$$

After matched filter being applied, the signal reached the radar can be denoted as, 


$$
v(t)=p(t)+J(t)=[c(t)+f(t)] * F^{-1}\left[|s(f)|^{2}\right]
$$

Where $F^{-1}\left[|s(f)|^{2}\right]$ is an kind of point spread function which has the envelope of $\operatorname{sinc}(\cdot)$ kind. The output signal after matched filtering is equivalent to the video function $f(t)$ with contrast of the target. So if we dynamically change the video function $f(t)$ in jamming signal pulse by pulse, a controlled effect could be reached in ISAR.

For the capability in 1-D and 2-D image of ISAR, the missile target can be viewed in many range cell and azimuth cell, it is necessary to change the video function in jamming signal according to the missile's posture so as to protect the missile target timely.

\subsection{Dynamic jamming against ISAR in 1-D image}

The 1-D image of ISAR is to get the position and intensity info of the missile target using the high resolution range profile. The response function equals to the sum of transmitted signals with a series of delay as blow,

$$
c(t)=\sum_{n=1}^{N} a_{n} \delta\left(t-\tau_{n}\right) .(6)
$$

As shown in (5) and its analysis, let the video function $f(t)$ be with the form,

$$
f(t)=\sum_{n=1}^{N} b_{n} \delta\left(t-\tau_{n}^{\prime}\right)
$$

So the output signal of the jamming also has the structure desired. If we change the video function pulse by pulse, in delay and intensity, the jamming effect could vary as expected.

\subsection{Dynamic jamming against ISAR in 2-D image}

The purpose of ISAR image is to get 2-dimentional distribution of $\mathrm{N}$-scatters of the target; the response function of such target is as blow,

$$
c\left(t_{r}, t_{a}\right)=\iint \sigma\left(t_{r}, t_{a}\right) \delta\left(t_{r}-\tau_{r}, t_{a}-\tau_{a}\right) d \tau_{r} d \tau_{a} .
$$


Where $t_{r}, t_{a}$ means the fast and slow sampling time.So, let the video function be with the form as below,

$$
f\left(t_{r}, t_{a}\right)=\iint \sigma_{j}\left(t_{r}, t_{a}\right) \delta\left(t_{r}-\tau_{r}, t_{a}-\tau_{a}\right) d \tau_{r} d \tau_{a}
$$

Meanwhile, let the jamming signals be with forms as blow,

$$
h\left(t_{r}, t_{a}\right)=h_{r}\left(t_{r}, t_{a}\right) \cdot h_{a}\left(t_{r}, t_{a}\right)(10)
$$

In order to get the processing gain in azimuth, it is necessary to add convolution modulation in azimuth, in which the reference function in azimuth is used. When different jamming templates are used in different processing aperture, varied image will be got in the ISAR.

Fig 1 shows the basic principle of different module in the jammer. The core idea of the jammer is to select varied template as the basic input to get the current needed template jamming image according to the posture between the missile and the ISAR, among which the range infois got by the launch timer in the missile, and the gesture of the missile can be got by the built-in posture computer in the missile. The signals are generated by phase modulation.

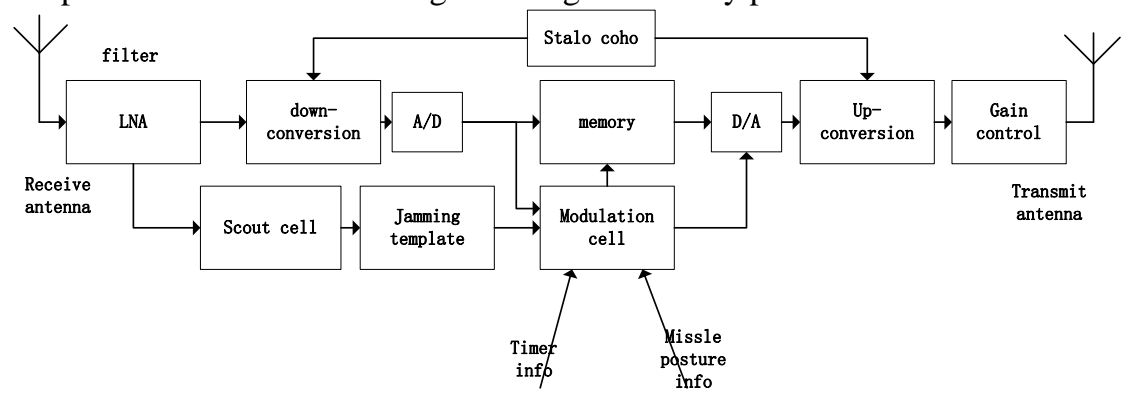

Fig 1 the block diagram of the jamming algorithm

Interpolation, at this time, plays a major role in the jamming templates generation at different angles according to the ISAR's LOS(look of sight),assume the varied templates of the missile at $\theta_{1}, \theta_{2}, \cdots, \theta_{N}$ are $T_{1}, T_{2}, \cdots, T_{N}$, as shown in fig 2.For the case that the target is at the LOS of $\theta_{k}$,where $\theta_{m} \leq \theta_{k} \leq \theta_{m+1}$, we get the jamming template $T_{k}$ by linear interpolation between the two adjacent templates as blow,

$$
T_{k}=T_{m}+\frac{\theta_{k}-\theta_{m}}{\theta_{m+1}-\theta_{m}} T_{m}
$$




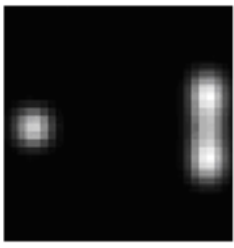

(a) $0^{0}$

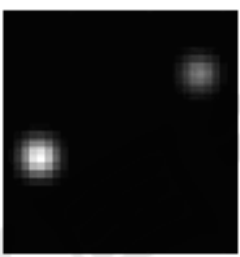

(b) $35^{0}$

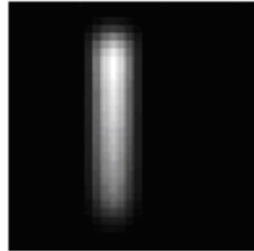

(c) $88^{\circ}$

Fig 2 the turntable image of the Agni missile model at different angles

\section{Analysis of the Performance of Jamming Algorithm}

\subsection{At the distal of the trajectory}

At this period, the missile has just entered into the trajectory, the distance to the ISAR is far, and jamming power is low. So it is suitable for the jamming strategy to select point template as the jamming patent. Fig3 shows the jamming effect. The envelope alignment cannot be finished, so it seems like noise jamming effect. The statistics of the correlation coefficient of the two adjacent range profile of the missile, as shown in fig 4 , shows that it changes from 0.97 to 0.16 , and it denotes that the point template is valid.
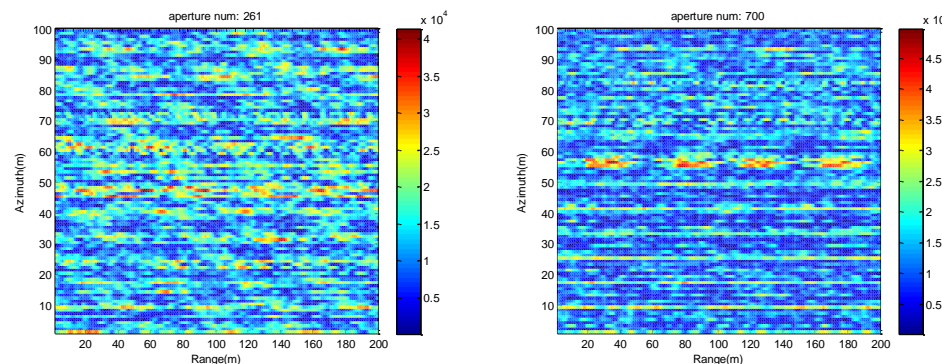

Fig 3 the jamming effect using point template at the distal of the trajectory

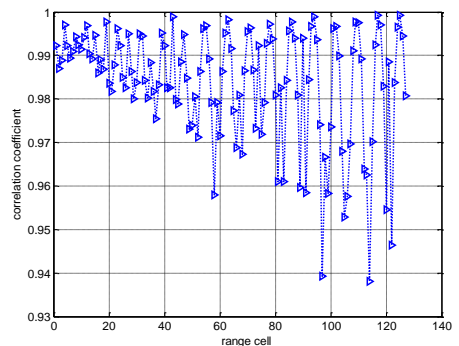

(a)Before jamming

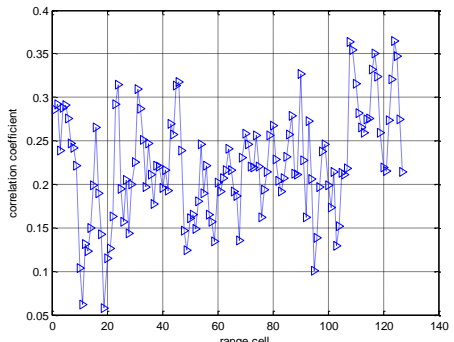

(b) after jamming

Fig 4 the correlation coefficient of the two adjacent range profile of the missile(at the distal) 


\subsection{At the proximal of the trajectory}

At this period, the missile target is near the ISAR and is of the risk on being detected and identified. So it is suitable for the jammer to select similar 1-D and 2-D image structure by interpolation between the closest templates. Fig5(a) shows the effect. Comparing with fig 4 (a),the statistics of the correlation coefficient of the two adjacent range profile of the missile, as shown in fig 5 (b), shows that it is near 0.99, and a false target appears with the true target. And it denotes that the interpolated template is valid.
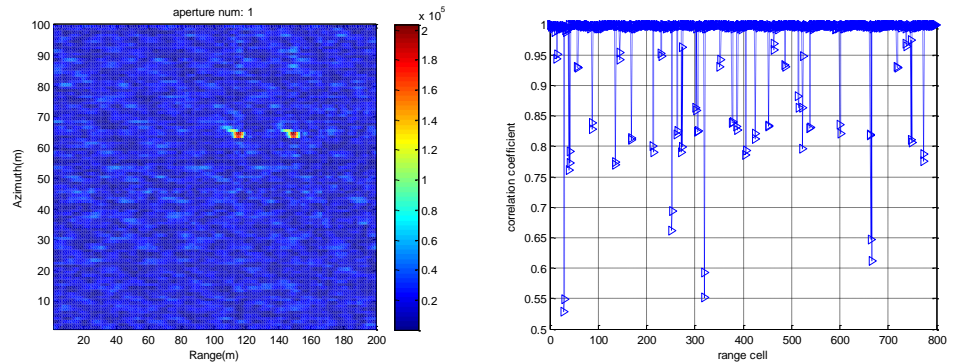

$\begin{array}{lll}\text { (a) jamming effect } & \text { (b) correlation coefficient of the two adjacent range profiles }\end{array}$ Fig5 simulation of the method at the proximal of the trajectory

\subsection{Analysis of the non-cooperation in bilateral time order}

The non-cooperation between jammer and the ISAR makes the issue that jamming signals cannot meet with the synthetic aperture time of the ISAR.

Firstly, when the jammer is working in the scouting time, the missile is of risk on being exposed before ISAR. Secondly, when the jammer scouts while jamming, analyzing the parameters of the LFM signals brings out great challenge.

We assign the jamming time order between scout and jamming as 1:9, 2:8 and $4: 6$, for the reason that the pulses needed for imaging are much more than those needed for scouting. And we get some results, from the statistical result, and it finds that the jamming effect, as shown in fig 5(a), changes dynamically and the jamming images are similar to the missile's posture.

\section{Conclusion}

A new active jamming method based on dynamic convolution against ISAR is proposed in this paper. There are also some short points for its large amount of calculation burden. Another choke point is that its data bandwidth request for there is posture info needing to transfer from the missile to the jammer. 


\section{Acknowledgment}

The authors would like to thank Dr Ning Tai who is with the Key Laboratory for ISAR signal processing, NUDT, for his good suggest in processing the model of Agni missile. They also wish to thank to the support by the National Natural Science Foundation of China (60802078), and the National Postdoctoral Science Foundation of China (20110491874).

\section{References}

1. M. J. Prickect, C. C. Chen. Principle of Inverse Synthetic Aperture Radar Imaging [M].EASCON record,1980,340 345

2. R. Z. Schneider and D. Fernandes, "Entropy Among a Sequency of SAR Images for Change Detection", Proceedings IGARSS 2003: 2623 2625,2013.

3. Li Song. A novel jamming Method for Dechirping ISAR based on the Micro-Doppler effect [J]. Joural of aerospace, 2012, vol.33 (6):736 745.

4. Xiaoyi. Pan, Wei. Wang, Jin. Liu, Dejun. Feng, Yi. Liu, and Guoyu. Wang, "Features extraction of rotationally symmetric ballistic targets based on micro-Doppler,' Progr. Electromagn Research,2013, vol.137(2):727 740,

5. Xiaoyi. Pan, Wei. Wang, Dejun. Feng, Yi. Liu, Qixiang.Fu, and Guoyu. Wang, "On deception jamming for countering bistatic ISAR based on subNyquist sampling,” IET Radar, Sonar Navigation,2014,vol.8(3):173 179.

6. Wang Kun. Research on M-Sequences Phase-Modulated Jamming to ISAR Imaging System [J]. Electrical engineering of Ships, 2012, vol.32 (5):58 60.

7. Li Jiangyuan, Wang Jianguo. A novel similar clutter jamming (SCJ) method to high-resolution SAR. Proceedings of 2006 CIE International Conference on Radar, 2006, vol.2:1070 1073.

8. P. E. Pace, D.J. Fouts, S. Ekestorm, and C. Karow, "Digital false-target image synthesiser for countering ISAR,” IEE Proc. Radar Sonar Navigat, 2002, vol.149(5):248 257, Oct. 